\title{
Oxygen Adsorption Induced Superconductivity in Ultrathin FeTe Film on $\mathrm{SrTiO}_{3}(001)$
}

\author{
Wei Ren ${ }^{1}$, Hao Ru ${ }^{2}$, Kun Peng ${ }^{3}$, Huifang $\mathrm{Li}^{3}{ }^{3}$, Shuai Lu ${ }^{3}$, Aixi Chen ${ }^{3}$, Pengdong Wang ${ }^{3}$, Xinwei Fang ${ }^{3}$, \\ Zhiyun $\mathrm{Li}^{3}$, Rong Huang ${ }^{3}$, Li Wang ${ }^{3, *}$, Yihua Wang ${ }^{2,4, *}$ and Fangsen $\mathrm{Li}^{1,3, * \mathbb{D}}$
}

1 School of Nano-Tech and Nano-Bionics, University of Science and Technology of China, Hefei 230026, China; wren2019@sinano.ac.cn

2 Department of Physics and State Key Laboratory of Surface Physics, Fudan University, Shanghai 200438, China; hru16@fudan.edu.cn

3 Vacuum Interconnected Nanotech Workstation (Nano-X), Suzhou Institute of Nano-Tech and Nano-Bionics (SINANO), Chinese Academy of Sciences (CAS), Suzhou 215123, China; 18752018528@163.com (K.P.); 51171213005@stu.ecnu.edu.cn (H.L.); slu2018@sinano.ac.cn (S.L.); axchen2017@sinano.ac.cn (A.C.); pdwang2020@sinano.ac.cn (P.W.); xwfang2020@sinano.ac.cn (X.F.); zhiyunli2018@sinano.ac.cn (Z.L.); rhuang2009@sinano.ac.cn (R.H.)

4 Shanghai Research Center for Quantum Sciences, Shanghai 201315, China

* Correspondence: lwang2017@sinano.ac.cn (L.W.); wangyhv@fudan.edu.cn (Y.W.); fsli2015@sinano.ac.cn (F.L.)

check for updates

Citation: Ren, W.; Ru, H.; Peng, K.; Li, H.; Lu, S.; Chen, A.; Wang, P.; Fang, X.; Li, Z.; Huang, R.; et al. Oxygen Adsorption Induced Superconductivity in Ultrathin $\mathrm{FeTe}$ Film on $\mathrm{SrTiO}_{3}(001)$. Materials 2021, 14, 4584. https://doi.org/10.3390/ ma14164584

Academic Editor:

Gueorgui Gueorguiev

Received: 21 July 2021

Accepted: 10 August 2021

Published: 15 August 2021

Publisher's Note: MDPI stays neutral with regard to jurisdictional claims in published maps and institutional affiliations.

Copyright: (c) 2021 by the authors. Licensee MDPI, Basel, Switzerland. This article is an open access article distributed under the terms and conditions of the Creative Commons Attribution (CC BY) license (https:/ / creativecommons.org/licenses/by/ $4.0 /)$.
Abstract: The phenomenon of oxygen incorporation-induced superconductivity in iron telluride $\left(\mathrm{Fe}_{1+\mathrm{y}} \mathrm{Te}\right.$, with antiferromagnetic (AFM) orders) is intriguing and quite different from the case of FeSe. Until now, the microscopic origin of the induced superconductivity and the role of oxygen are far from clear. Here, by combining in situ scanning tunneling microscopy/spectroscopy (STM/STS) and X-ray photoemission spectroscopy (XPS) on oxygenated FeTe, we found physically adsorbed $\mathrm{O}_{2}$ molecules crystallized into $c(2 / 3 \times 2)$ structure as an oxygen overlayer at low temperature, which was vital for superconductivity. The $\mathrm{O}_{2}$ overlayer were not epitaxial on the FeTe lattice, which implied weak $\mathrm{O}_{2}-$ FeTe interaction but strong molecular interactions. The energy shift observed in the STS and XPS measurements indicated a hole doping effect from the $\mathrm{O}_{2}$ overlayer to the FeTe layer, leading to a superconducting gap of $4.5 \mathrm{meV}$ opened across the Fermi level. Our direct microscopic probe clarified the role of oxygen on FeTe and emphasized the importance of charge transfer effect to induce superconductivity in iron-chalcogenide thin films.

Keywords: FeTe; oxygen incorporation; superconductivity; microscopic origin

\section{Introduction}

Iron-chalcogenide $\mathrm{Fe}(\mathrm{Se}, \mathrm{Te})$ superconductors are an important family of $\left(T_{\mathrm{c}}\right)$ ironbased high transition temperature $\left(T_{c}\right)$ superconductors. The monolayer grown on $\mathrm{SrTiO}_{3}$ (STO) still holds the record in $T_{\mathrm{c}}$ with much-enhanced superconducting pairing [1-3]; the strong spin-orbit coupling in Te-alloyed compounds leads to a topological band structure [4-7], the family also exhibits rich magnetic phases strongly dependent on the chemical composition [8,9]. Non-superconducting FeTe with antiferromagnetic (AFM) orders was considered as the parent compound of Iron-chalcogenide superconductors [10]. Superconductivity could be induced in FeTe through Se or S substitution [10] and oxygen incorporation by low-temperature annealing or long-time exposure in an $\mathrm{O}_{2}$ atmosphere [11-21]. Despite lots of efforts towards understanding oxygenated FeTe bulk crystals [15-22] and thin films [11-14,21], the microscopic role played by oxygen is still unknown.

Zheng et al. [12] found that interstitial oxygen, rather than oxygen substitution [23], was responsible for the emergence of superconductivity. The interstitial oxygen $[13,16]$ would dope holes into FeTe [17], suppress the AFM ordering and then induce superconductivity. Yamazaki et al. [19] and Sun et al. [18] have studied the dynamics of oxygen annealing and concluded that the superconductivity first emerged on the surface and the 
superconducting regions moved inside with non-superconducting materials left on the surface, such as $\mathrm{Fe}_{2} \mathrm{O}_{3}, \mathrm{TeO}_{x}, \mathrm{FeTe}_{2}$. The controversial results from different experimental probes call for a unified study of the crystal, chemical and electronic structures on thin films with controlled oxygen incorporation. However, this is generally challenging to achieve for ex situ measurements because samples exposed to the atmosphere inevitably change surface morphology and chemistry. In this study, we overcame this challenge by integrating one ultra-high vacuum environment for the sample growth and oxygenation process along with spectroscopic tools with elemental and spatial resolution.

First, we grew nearly stoichiometric 10 Unit-Cell (UC) FeTe epitaxy ultrathin films by molecular beam epitaxy (MBE) and then studied the adsorption of $\mathrm{O}_{2}$ incorporation by combined in situ STM and X-ray photoelectron spectroscopy (XPS). We found that $\mathrm{O}_{2}$ molecules physically adsorbed on FeTe surface under low $\mathrm{O}_{2}$ partial pressure at room temperature and crystallized into $c(2 / 3 \times 2)$ structure, which gradually disappeared after heating up to $100{ }^{\circ} \mathrm{C}$ in vacuum. No surface oxidation can be found. The band shift observed in STS and XPS suggests hole doping from the $\mathrm{O}_{2}$ overlayer to FeTe, which induced a superconducting gap in FeTe. It agrees with the ex situ transport measurement that long-time exposure to air can induce superconducting transition in 10UC FeTe on STO.

\section{Materials and Methods}

High-quality 10UC FeTe thin films were grown on $\mathrm{SrTiO}_{3}(001)$ by using a Unisoku ultrahigh vacuum (UHV) low-temperature STM system (Unisoku 1300 9T-2T-2T, 2016, Hirakata, Osaka, Japan), equipped with an MBE chamber for film preparation. The base pressure of our MBE chamber is $1 \times 10^{-10} \mathrm{mbar}$ and does not exceed $5 \times 10^{-10} \mathrm{mbar}$ during FeTe epitaxial growth. The Nb doped-STO substrate (Nb: 0.7\% wt, KMT comp.) was firstly annealed at $1000{ }^{\circ} \mathrm{C}$ for $1 \mathrm{~h}$ and then kept at $280^{\circ} \mathrm{C}$ during FeTe growth. High purity $\mathrm{Fe}(99.9999 \%)$ and $\mathrm{Te}(99.9999 \%)$ sources were co-evaporated onto $\mathrm{SrTiO}_{3}(001)$ substrates from two standard Knudsen cells. To improve the quality of crystallization, FeTe films were annealed at $280^{\circ} \mathrm{C}$ for $1 \mathrm{~h}$ after growth. The $\mathrm{O}_{2}$ gas (99.999\%) was introduced to a small preparation chamber with a base pressure of $2 \times 10^{-9} \mathrm{mbar}$. Then the oxygen absorption was carried out at room temperature under $\mathrm{O}_{2}$ partial pressure of $1.6 \times 10^{-4} \mathrm{mbar}$ (if not specified otherwise). After $\mathrm{O}_{2}$ absorption, the samples were in situ transferred to the STM chamber or XPS equipment for further characterizations. For electronic transport measurement, 10UC FeTe films were grown on high-resistance STO and covered with the Te protection layer.

All STM/STS measurements were performed at a temperature of $4.7 \mathrm{~K}$ with a bias voltage applied to the sample. Polycrystalline $\mathrm{Pt} / \mathrm{Ir}$ tips were cleaned by electron bombardment and then carefully calibrated on Ag films grown on Si (111). All STM topographic images were obtained under a constant current mode. The differential conductance $\mathrm{d} I / \mathrm{d} V$ spectra were acquired by using a standard lock-in technique at a frequency of $983 \mathrm{~Hz}$. The STM topographic images were processed with WSXM software (WSxM 5.0 Develop 10.0, 2020, Julio Gómez Herrero \& José María Gómez Rodríguez, Madrid, Spain) [24].

After STM/STS measurements, the samples were transferred via a UHV interconnection pipeline in Nano-X for XPS/UPS characterization without exposure to air. XPS/UPS experiments were carried out by using a commercial analyzer (PHI 5000, VersaProbe, ULVAC-PHI, $\sim 5 \times 10^{-10}$ mbar, 2016, Chigasaki, Kanagawa, Japan), equipped with a monochromatic $\mathrm{Al} \mathrm{K} \alpha$ X-ray source of $1486.7 \mathrm{eV}$. The binding energy (BE) of core-level peaks were calibrated with respect to the $\mathrm{C}-\mathrm{C} 1 \mathrm{~s}$ bond $(\mathrm{BE}=284.8 \mathrm{eV})$. A monochromatized He I with an energy of $21.22 \mathrm{eV}$ were used for UPS measurement. During the UPS measurement, a voltage of $-5 \mathrm{~V}$ was applied between the sample and the spectrometer.

\section{Results}

High-quality FeTe films are epitaxially grown on STO substrates via step flow growth mode. Figure 1a shows the typical surface morphology of 10UC FeTe film, and Figure 1b shows the atomically resolved image with Te termination. Fast Fourier transform image 
yields enlarged lattice constant of $\sim 0.384 \mathrm{~nm}$, similar to the case of FeSe on STO [1]. No Fe adatoms (or interstitial Fe atoms) and Te vacancies can be observed on the surface, which indicates nearly stoichiometric FeTe epitaxy films. To carry out electronic transport measurement, the protection Te layer was deposited on the 10UC FeTe surface, and the results were shown in Figure 1c. No superconducting transition but semiconducting behavior was observed in initially grown 10UC FeTe film down to $1.8 \mathrm{~K}$, consistent with previous experiments $[25,26]$. Compared with bulk or thick FeTe films $[11,13,23,27-29]$, we have not found an anomaly in the $R$ - $T$ curve between $40 \mathrm{~K}$ and $80 \mathrm{~K}$, which further demonstrates free of Fe interstitial atoms [26]. AFM state may be suppressed here. After exposing the sample to air for 8 days, the resistance turned downward at $\sim 3.2 \mathrm{~K}$, as shown in the inset of Figure 1c. Considering the fact of long-time exposure-induced superconductivity in FeTe compounds, such downward should be attributed to superconducting transition. It is further verified by the suppression of transition under external magnetic fields in Figure 1d, characteristic of superconductivity. Non-zero resistance here suggests the developed superconductivity is not uniform. We found that exposure to air for a longer time would increase the superconducting transition temperature further.
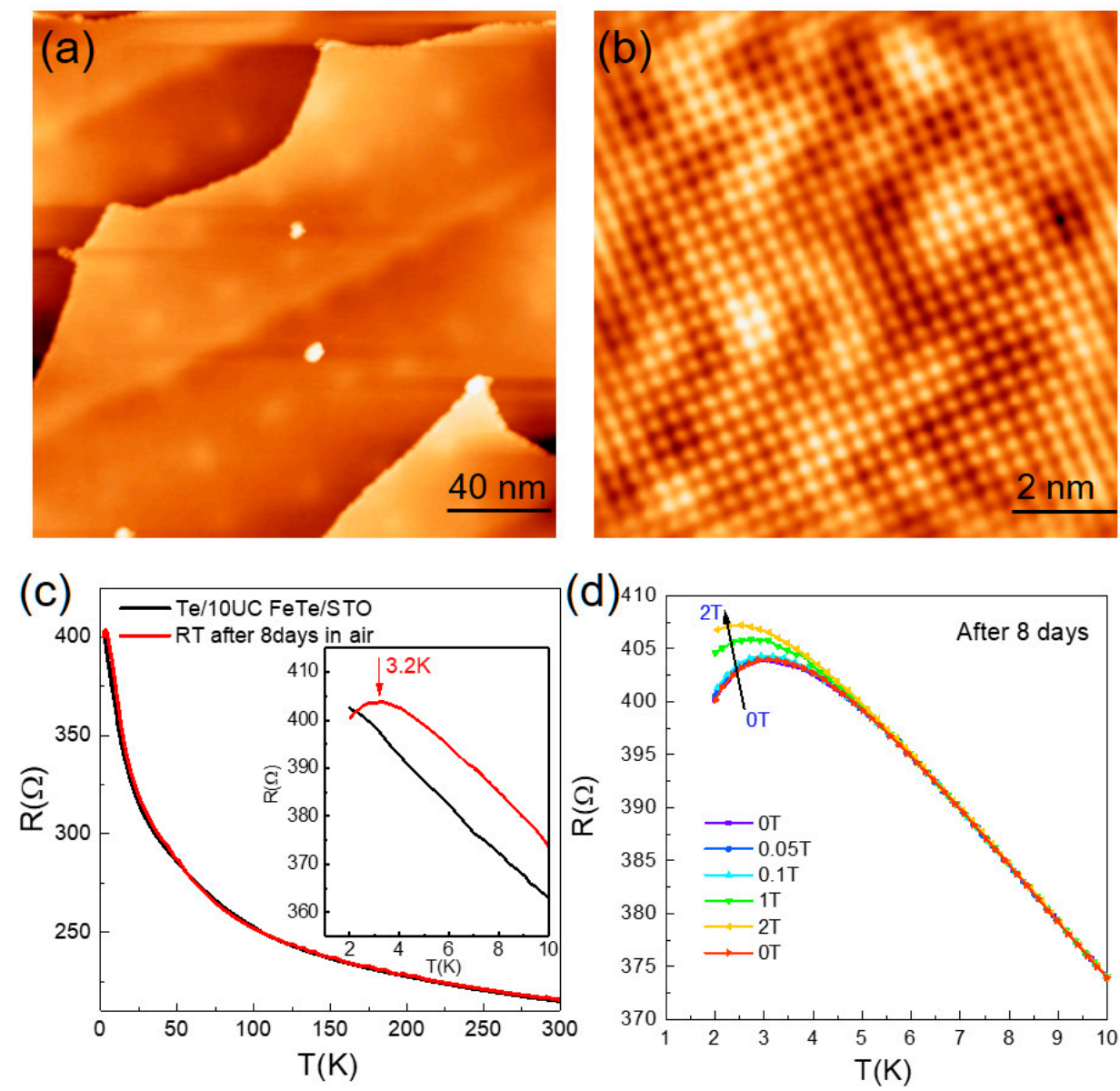

Figure 1. (Color online) (a) Surface topography $(200 \mathrm{~nm} \times 200 \mathrm{~nm})$ of the 10UC FeTe films on the Nb:STO. Scanning condition: sample bias $V_{\mathrm{s}}=3 \mathrm{~V}$, tunneling current $I_{t}=100 \mathrm{pA}$. (b) Typical atomically resolved STM image $(10 \mathrm{~nm} \times 10 \mathrm{~nm})$ of 10UC FeTe. Scanning condition: sample bias $V_{\mathrm{s}}=0.7 \mathrm{~V}$, tunneling current $I_{t}=200 \mathrm{pA}$. (c) Temperature-dependent resistance $(R-T)$ curve of 10UC FeTe thin film on HR-STO before and after exposure to air for 8 days. Inset: $R-T$ curve under low temperature, showing turn-down behavior after exposure to air. (d) Temperature-dependent resistance of 10UC FeTe after exposure to air for 8 days under an out-of-plane magnetic field up to $2 \mathrm{~T}$. The turn-down behavior was suppressed under magnetic fields and recovered after magnetic field withdrawal. 
To figure out the superconducting mechanism in FeTe after exposure to $\mathrm{O}_{2}$, we carried out the oxygenated process under a well-control condition and probed the atomic and electronic structures microscopically. Figure 2a shows the surface morphology of the initial adsorption stage after exposure 10UC FeTe to $\mathrm{O}_{2}$ under a partial pressure of $1.6 \times 10^{-4} \mathrm{mbar}$ for $15 \mathrm{~min}$. We can observe extra layers along the step edge, while other uncovered clean FeTe areas still remain unchanged, as illustrated by the atomically resolved STM image in the inset. No $\mathrm{O}_{\mathrm{Te}}$ substitution or interstitial $\mathrm{O}$ atoms can be observed. The enlarged image in Figure $2 \mathrm{~b}$ shows there are small compacted islands with a height of $\sim 0.18 \mathrm{~nm}$ in the overlayer, as shown in Figure 2c. Some dispersed clusters can be also found at the domain boundaries, which should be the ones that have not coarsened into the islands. If further increasing the amount of inlet $\mathrm{O}_{2}$, nearly the whole surface can be covered by the adsorbed overlayers, as shown in Figure S1a. The uncovered areas are still very clean in Figure S1b.
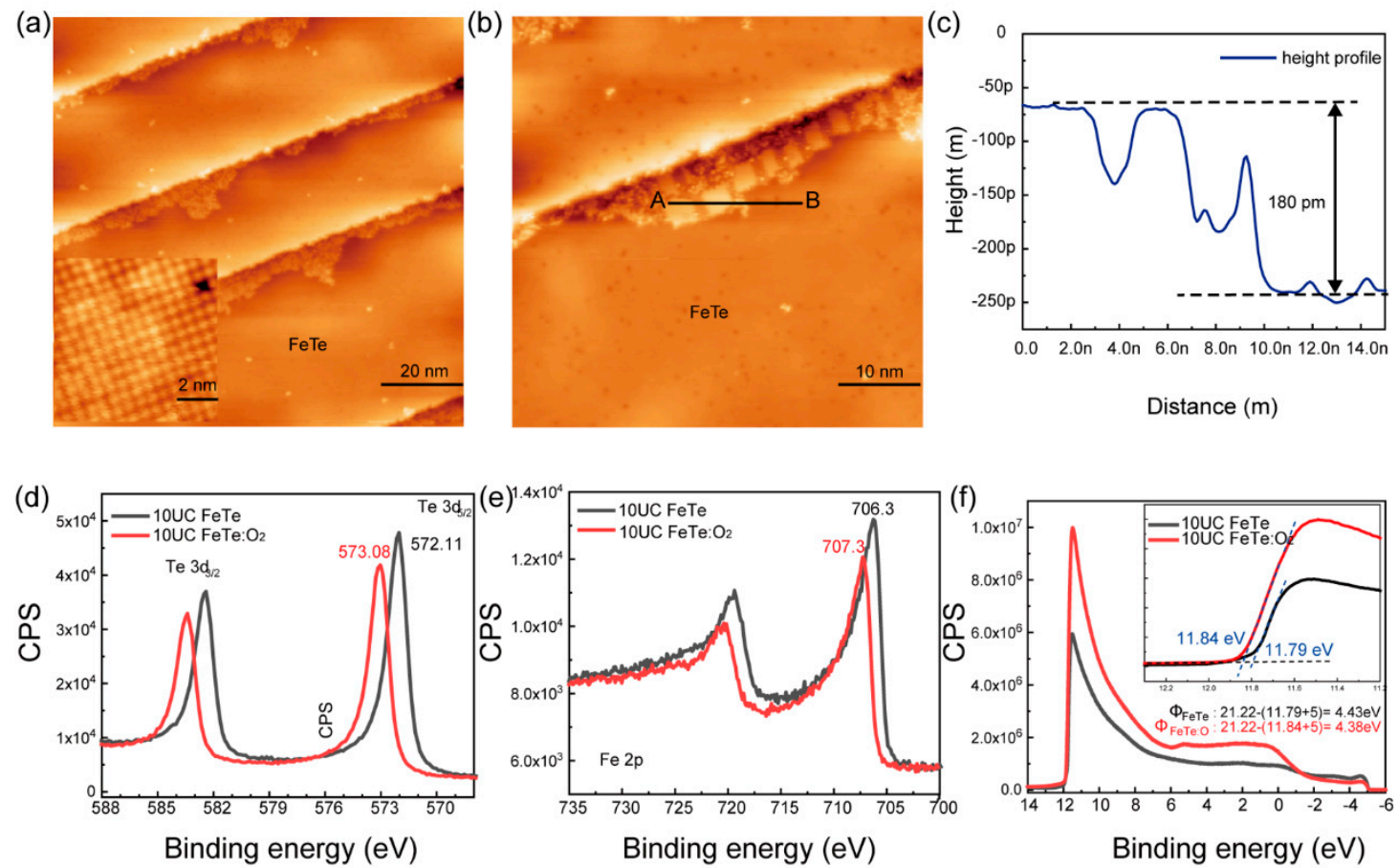

Figure 2. (Color online) (a) Surface topography $(100 \mathrm{~nm} \times 100 \mathrm{~nm})$ of the 10UC FeTe films after exposure to oxygen partial pressure of $1.6 \times 10^{-4}$ mbar for $15 \mathrm{~min}$ at RT. Scanning condition: sample bias $V_{\mathrm{s}}=1 \mathrm{~V}$, tunneling current $I_{t}=100 \mathrm{pA}$. $\mathrm{O}_{2}$ overlayers were initially formed along the step edge. Inset is the atomically resolved STM image of the uncovered clean FeTe surface. $\left(\right.$ b) Enlarged STM image $(50 \mathrm{~nm} \times 50 \mathrm{~nm})$ of $\mathrm{O}_{2}$ overlayers $\left(\mathrm{FeTe}: \mathrm{O}_{2}\right)$ on FeTe surface. Scanning condition: sample bias $V_{\mathrm{s}}=0.50 \mathrm{~V}$, tunneling current $I_{t}=100 \mathrm{pA}$. (c) The height profile of FeTe : $\mathrm{O}_{2}$ along line AB in panel b. (d,e) XPS core-level spectra of $\mathrm{Te} 3 \mathrm{~d}(\mathbf{d})$ and $\mathrm{Fe} 2 \mathrm{p}(\mathbf{e})$ on as-prepared $10 \mathrm{UC}$ FeTe and FeTe : $\mathrm{O}_{2}$ covered surface, respectively. No trance of $\mathrm{Te}-\mathrm{O}$ and Fe-O signals can be observed. The spectra show apparent peak shifts after oxygen adsorption. (f) UPS spectra of 10UC FeTe covered $w / o$ FeTe $: \mathrm{O}_{2}$, and the added voltage here is $-5 \mathrm{~V}$. Inset is the large view.

To unravel the nature of adsorbed overlayer $\left(\mathrm{O}_{2}\right.$ molecules, $\mathrm{FeO}_{\mathrm{x}}, \mathrm{TeO}_{\mathrm{x}}, \mathrm{Fe}-\mathrm{Te}-\mathrm{O}$ or others?), Figure 2d,e present the core-level XPS spectra of Te $3 \mathrm{~d}$ and $\mathrm{Fe} 2 \mathrm{p}$ before and after inlet $\mathrm{O}_{2}$, respectively. After inlet $\mathrm{O}_{2}$ under a partial pressure of $1.6 \times 10^{-4} \mathrm{mbar}$, we do not observe Te-O and Fe-O spectra signals. If exposure to $\mathrm{O}_{2}$ partial pressure of $1.5 \times 10^{-1}$ mbar for $20 \mathrm{~min}$, as shown in Figure S3c clear Te-O core level peak can be identified, which suggests surface oxidation under high $\mathrm{O}_{2}$ partial pressure. The XPS spectra of Te $3 \mathrm{~d}$ and Fe $2 \mathrm{p}$ show an obvious peak shift toward higher binding energy, which hints hole doping effect in FeTe. The overlayer would gradually desorb when heating up to $100{ }^{\circ} \mathrm{C}$ and disappeared after annealing at $200{ }^{\circ} \mathrm{C}$ for $2 \mathrm{~h}$. Only a clean FeTe surface was left. The overlayer will re-adsorb after inlet $\mathrm{O}_{2}$ again. Thus, we can rule out the 
possibility of Fe- or Te-related compounds because there was negligible residual $\mathrm{Fe}$ or $\mathrm{Te}$ atoms after growth. In addition, we notice there are some dispersed clusters higher than the compacted islands in Figures $2 \mathrm{~b}$ and $3 \mathrm{a}$, which helps us to conclude that the overlayer should be $\mathrm{O}_{2}$ molecules rather than stacked $\mathrm{O}$ atoms. The shape of $\mathrm{O} 1 \mathrm{~s}$ core level almost remains unchanged after $\mathrm{O}_{2}$ adsorption under a partial pressure of $1.6 \times 10^{-4} \mathrm{mbar}$, as illustrated in Figure S3, but changes a lot after surface oxidation due to the signal from $\mathrm{O}-\mathrm{Te}$ and O-Fe. It implies the signal of $\mathrm{O} 1 \mathrm{~s}$ at $529.5 \mathrm{eV} \sim 531 \mathrm{eV}$ are mainly from the STO substrate, which, as expected, exhibits a similar core level shift as Te $3 \mathrm{~d}$, as shown in Figure S3a. It also consists of the nature of physically adsorbed $\mathrm{O}_{2}$ molecules, where characteristic core-level XPS peak always cannot be found. Thus, we name the overlayer heterostructure as $\mathrm{FeTe}: \mathrm{O}_{2}$. UPS measurements in Figure $2 \mathrm{f}$ show that the work function just slightly changed from $\phi_{\mathrm{FeTe}} \sim 4.43 \mathrm{eV}$ to $\phi_{\mathrm{FeTe}} \mathrm{O}_{2} \sim 4.38 \mathrm{eV}$ after $\mathrm{O}_{2}$ adsorption. The small difference in work function is also consistent with the nature of $\mathrm{O}_{2}$ molecules in the overlayer.
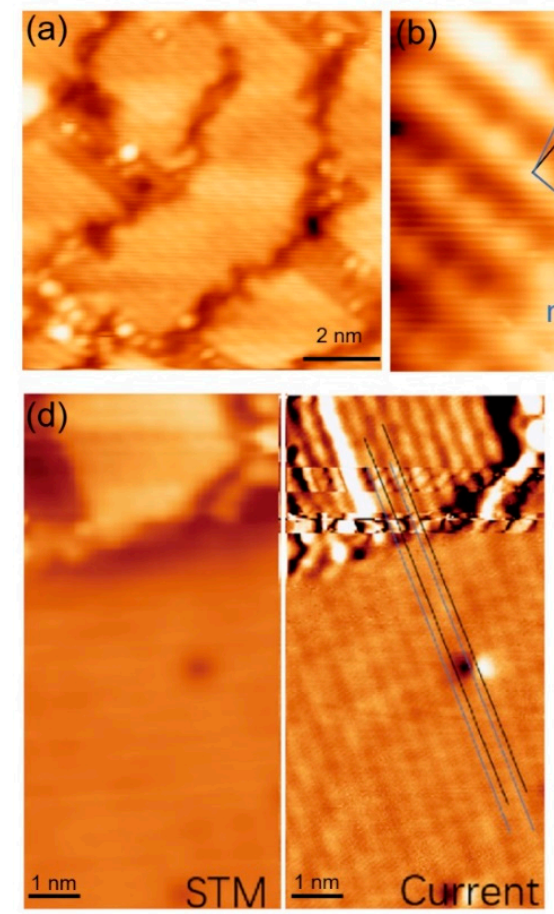
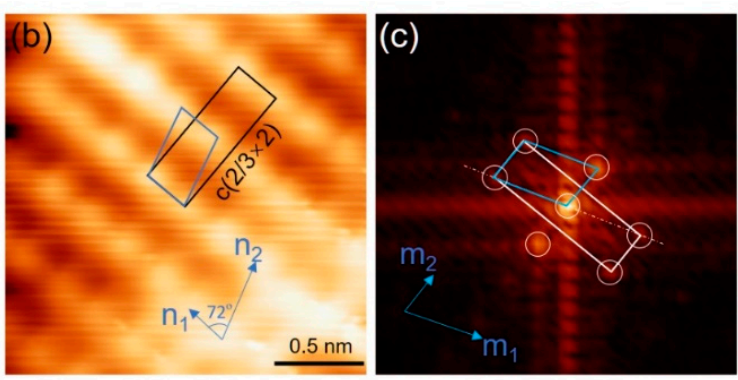

(e)

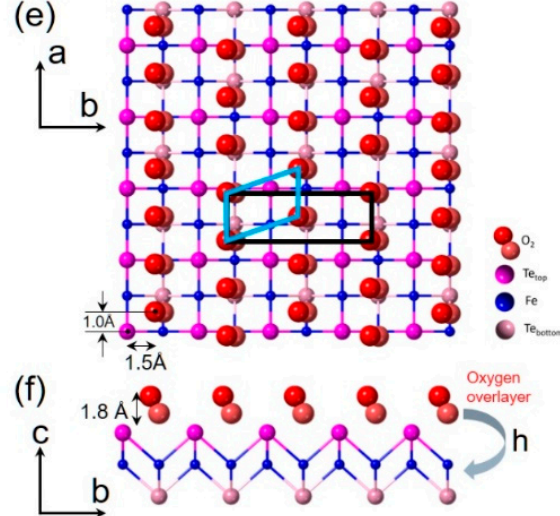

Figure 3. (Color online) (a) Small compacted FeTe : $\mathrm{O}_{2}$ islands on 10UC FeTe. Dispersed clusters can be observed at domain boundaries. Scanning condition: sample bias $V_{\mathrm{s}}=1 \mathrm{~V}$, tunneling current $I_{t}=200$ pA. (b) Atomically resolved STM images $(2 \mathrm{~nm} \times 2 \mathrm{~nm})$ on FeTe : $\mathrm{O}_{2}$ islands. Scanning condition: sample bias $V_{\mathrm{s}}=0.1 \mathrm{~V}$, tunneling current $I_{t}=200 \mathrm{pA}$. (c) The fast Fourier transform image of (b). White circles mark the reciprocal lattice. (d) STM topographic (left panel) and constant current (right panel) images $(5 \mathrm{~nm} \times 10 \mathrm{~nm})$ of the $10 \mathrm{UC}$ FeTe films with one $\mathrm{FeTe}: \mathrm{O}_{2}$ island. The current image helps us to measure the adsorption geometrical configuration. Scanning condition: sample bias $V_{\mathrm{s}}=0.3 \mathrm{~V}$, tunneling current $I_{t}=300 \mathrm{pA}$. (e,f) Schematic diagrams of geometrical configuration of $\mathrm{O}_{2}$ adsorption overlayer on FeTe: (e) top side and (f) side views.

Figure 3a shows some small compacted $\mathrm{FeTe}: \mathrm{O}_{2}$ islands, where striped structures can be clearly observed. Only two perpendicular orientations were distinguished, which consist of the tetragonal symmetry of the underlying FeTe lattice. The atomically resolved structure of adsorbed FeTe : $\mathrm{O}_{2}$ islands is shown in Figure 3b. The distance between neighboring bright rows is $\sim 0.386 \mathrm{~nm}$, nearly equal to the lattice constant $a_{\mathrm{FeTe}}$ of FeTe. The corresponding fast Fourier transform image in Figure $3 \mathrm{c}$ yields an in-plane lattice of $n_{1}$ with a value of $0.25 \pm 0.02 \mathrm{~nm}$ and $n_{2}$ with a value of $0.41 \pm 0.02 \mathrm{~nm}$. The angle between $n_{1}$ and $n_{2}$ is measured to be $\sim 72^{\circ}$. We notice that the spot distance of $0.25 \mathrm{~nm}$ along $n_{1}$ 
is nearly equal to $2 / 3 a_{\mathrm{FeTe}}$. Thus, the lattice can be notated as $c(2 / 3 \times 2)$, as shown in Figure $3 b$,c. Such lattice arrangement demonstrates a close relationship between the $\mathrm{O}_{2}$ adsorption overlayer $\left(\mathrm{FeTe}: \mathrm{O}_{2}\right)$ and the FeTe surface. To locate the precise adsorption sites of $\mathrm{O}_{2}$ on FeTe, we achieved an atomically resolved image on both the FeTe : $\mathrm{O}_{2}$ island and FeTe surface in Figure 3d. The results are shown in the structural model in Figure 3e. As illustrated, along the $n_{1}$ direction, the adsorption sites of $\mathrm{O}_{2}$ molecules are not well-defined relative to FeTe lattice and not at high-symmetry points. It not only signifies that the van der Waals interaction between $\mathrm{O}_{2}$ molecules plays a vital role in the overlayer but also verifies the absence of chemical bonding between the $\mathrm{O}_{2}$ interlayer and the FeTe surface.

To explore how the electronic structure changes due to $\mathrm{O}_{2}$ absorption, we conducted detailed differential conductance $\mathrm{d} I / \mathrm{d} V$ measurements, which are known to be proportional to the local density of state (LDOS). On as-prepared and uncovered clean 10UC FeTe surfaces, the STS remain nearly unchanged with a "V" shape across the Fermi level in Figure $4 a$, consistent with previous studies [2,30]. A hump at the negative bias voltage shift from $-0.37 \mathrm{~V}$ to $-0.325 \mathrm{~V}$ on FeTe : $\mathrm{O}_{2}$ after $\mathrm{O}_{2}$ adsorption, which indicates hole doping effect to $\mathrm{FeTe}$, agreeing well with the observation of peak shift in XPS experiments in Figure 2. Such oxygen-induced hole doping effect [31] was recently observed on the $\mathrm{CsV}_{3} \mathrm{Sb}_{5}$ with an increased superconducting transition temperature. Additionally, hole doping effects were observed in $\mathrm{Bi}_{2} \mathrm{Te}_{3} / \mathrm{FeTe}[32,33]$ and $\mathrm{Sb}_{2} \mathrm{Te}_{3} / \mathrm{FeTe}$ [26] heterostructures and thought to be the origin of two-dimensional superconductivity in the heterostructures. Figure $4 \mathrm{~b}$ shows the $\mathrm{d} I / \mathrm{d} V$ spectra in a small energy range. A clear $\mathrm{V}$-shape was observed on a clean FeTe surface, similar to the one on the monolayer FeTe [2] and thick FeTe film [30]. Strikingly, on FeTe : $\mathrm{O}_{2}$ islands two pronounced peaks with an energy gap of $\sim 9 \mathrm{meV}$ were clearly identified across the Fermi level. Considering the facts of previous observation of hole-doping-induced superconductivity in the $\mathrm{Bi}_{2} \mathrm{Te}_{3} / \mathrm{FeTe}$ heterostructure and the superconductivity observed in 10UC FeTe/STO sample after long-time exposure to air, we conclude that the gap on FeTe : $\mathrm{O}_{2}$ is just a superconducting gap with $\Delta \sim 4.5 \mathrm{meV}$, larger than the one on $\mathrm{Bi}_{2} \mathrm{Te}_{3} / \mathrm{FeTe}$ heterostructure $(\sim 2.5 \mathrm{meV})$ [32,34]. It should be due to the interfacial effect on STO substrate because similar enhanced superconductivities have been observed on the $\mathrm{Pb}$ islands [35] and monolayer $\mathrm{FeTe}_{x} \mathrm{Se}_{1-\mathrm{x}}[1,2,25]$. We have studied the spatial distribution of the superconducting gap on the $\mathrm{FeTe}: \mathrm{O}_{2}$ islands in Figure 4c. Pronounced coherent peaks can be observed in all the $\mathrm{d} I / \mathrm{d} V$ curves.
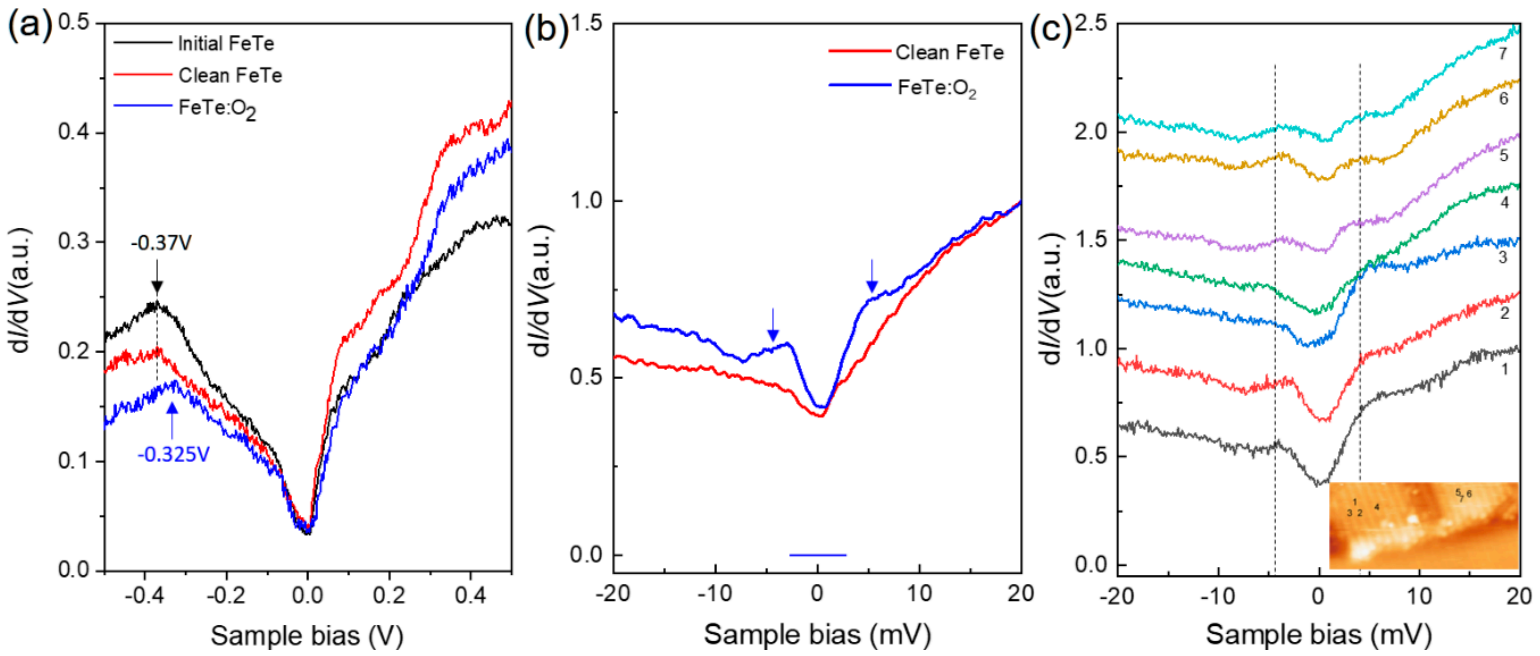

Figure 4. (Color online) (a) Typical $\mathrm{d} I / \mathrm{d} V$ spectra on 10UC FeTe before and after $\mathrm{O}_{2}$ adsorption. A hump at negative energy shows a clear shift on FeTe : $\mathrm{O}_{2}$ overlayer. Starting condition: $V_{\mathrm{s}}=0.5 \mathrm{~V}$, tunneling current $I_{t}=100 \mathrm{pA}$. (b) The zoomed-in $\mathrm{d} I / \mathrm{d} V$ spectra on clean FeTe area and $\mathrm{FeTe}: \mathrm{O}_{2}$ overlayer. An energy gap with two pronounced peaks was observed across the Fermi level, which is a superconducting gap. Starting condition: $V_{\mathrm{s}}=20 \mathrm{mV}$, tunneling current $I_{t}=200 \mathrm{pA}$. (c) A series of $\mathrm{d} I / \mathrm{d} V$ spectra on FeTe : $\mathrm{O}_{2}$, showing the energy gap can be observed on the whole FeTe : $\mathrm{O}_{2}$ islands. Starting condition: $V_{\mathrm{s}}=20 \mathrm{mV}$, tunneling current $I_{t}=200 \mathrm{pA}$. 
The observation of enhanced superconducting gap on FeTe : $\mathrm{O}_{2}$ islands is intriguing and distinct from all the previously proposed mechanisms. Physically adsorbed $\mathrm{O}_{2}$ molecules rather than interstitial oxygen or oxygen substitution are responsible for superconductivity in oxygenated FeTe. Previously, annealing under an elevated temperature or quite a long-time aging may be aimed at the formation of a uniform $\mathrm{O}_{2}$ overlayer into the FeTe layer. Our study here also emphasizes the importance of the hole doping effect and interfacial effect to induce superconductivity on FeTe. We have not observed a superconducting gap on FeTe: $\mathrm{O}_{2}$ overlayer on monolayer FeTe, which may be due to the extra electron doping from the STO substrate.

\section{Conclusions}

In summary, we have systematically investigated the changes of morphology and electronic structure of 10UC FeTe films after $\mathrm{O}_{2}$ adsorption. Ex-situ transport measurements demonstrate long-term exposure to air could induce superconducting transition in 10UC FeTe ultrathin film, which verifies the effect of oxygen incorporation. Initially, $\mathrm{O}_{2}$ molecules physically adsorbed on the FeTe surface and formed compacted FeTe : $\mathrm{O}_{2}$ islands with $c(2 / 3 \times 2)$ structure at low temperatures. No oxygen-related defects can be detected on the uncovered clean FeTe surface. Energy shifts in STS and XPS measurements point out obvious hole doping into FeTe from the oxygen overlayer. Such hole doping results in a superconducting gap of $\sim 4.5 \mathrm{meV}$ opened with apparent coherent peaks. Our study here directly provides microscopical insights about the role of oxygen on FeTe thin film.

Supplementary Materials: The following are available online at https:/ / www.mdpi.com/article/10

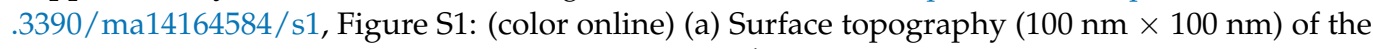
10UC FeTe films after exposure to $\mathrm{O}_{2}$ under $1.6 \times 10^{-4} \mathrm{mbar}$ for $4 \mathrm{~h}$ at room temperature. Scanning condition: Vs $=3 \mathrm{~V}$, It $=100 \mathrm{pA}$. (b) Enlarged view $(50 \mathrm{~nm} \times 50 \mathrm{~nm})$ of surface topography, showing clean FeTe surface with $\mathrm{O}_{2}$ overlayer. Scanning condition: Vs $=1 \mathrm{~V}$, It $=100 \mathrm{pA}$, Figure S2: (color online) The XPS survey scan on $10 \mathrm{UC}$ FeTe after $\mathrm{O}_{2}$ adsorption, showing no other impurities can be observed after $\mathrm{O}_{2}$ inlet, Figure S3: (color online) (a) The XPS core level spectra of O1s before and after $\mathrm{O}_{2}$ inlet, showing similar shift as Te $3 \mathrm{~d}$ and Fe 2p. (b,c) The normalized and shifted XPS core level spectra of $\mathrm{O} 1 \mathrm{~s}$ (b) and Te 3d (c) on $10 \mathrm{UC}$ FeTe before and after $\mathrm{O}_{2}$ adsorption, showing the shapes of $\mathrm{O} 1 \mathrm{~s}$ or Te 3d are nearly overlapped. We also added the XPS spectra on the oxidized FeTe/STO when exposure to $\mathrm{O}_{2}$ partial pressure of $1.5 \times 10^{-1} \mathrm{mbar}$ for $20 \mathrm{~min}$.

Author Contributions: Conceptualization, F.L. and Y.W.; methodology, F.L. and W.R.; software, W.R.; validation, W.R., H.R. and K.P., H.L., S.L., A.C., P.W., X.F., Z.L., R.H. and L.W.; formal analysis, F.L., W.R., H.R. and Y.W.; investigation, F.L. and W.R.; resources, F.L. and Y.W.; data curation, F.L. and W.R.; writing—original draft preparation, F.L. and W.R.; writing—review and editing, F.L. and W.R.; visualization, F.L. and W.R.; supervision, F.L. and Y.W.; project administration, F.L. and Y.W.; funding acquisition, F.L. and Y.W. All authors have read and agreed to the published version of the manuscript.

Funding: This work is supported by the National Natural Science Foundation of China (Grants No. 11604366, No. 11634007). F. Li acknowledges support from the Youth Innovation Promotion Association of the Chinese Academy of Sciences (2017370). Y. Wang would like to acknowledge partial support by the Ministry of Science and Technology of China under Grant numbers 2017YFA0303000 and 2016YFA0301002, NSFC Grant No. 11827805, and Shanghai Municipal Science and Technology Major Project Grant No.2019SHZDZX01.

Institutional Review Board Statement: Not applicable.

Informed Consent Statement: Not applicable.

Data Availability Statement: The data presented in this study are available on request from the corresponding authors.

Acknowledgments: We thank Xu-cun Ma, Li-li Wang and Haiping Lin for helpful discussion.

Conflicts of Interest: The authors declare that they have no conflict of interest. 


$\begin{array}{ll}\text { Abbreviations } \\ \text { AFM } & \text { Antiferromagnetic } \\ \text { UC } & \text { Unit-Cell } \\ \text { UHV } & \text { Ultrahigh Vacuum } \\ \text { BE } & \text { Binding Energy } \\ \text { LDOS } & \text { Local Density of State }_{\text {STO }} \text { SrTiO }_{3} \\ \text { STM } & \text { Scanning Tunneling Microscopy }_{\text {STS }} \text { Scanning Tunneling Spectroscopy } \\ \text { XPS } & \text { X-ray Photoelectron Spectroscopy } \\ \text { UPS } & \text { Ultraviolet Photoelectron Spectrometer } \\ \text { MBE } & \text { Molecular Beam Epitaxy }\end{array}$

\section{References}

1. Wang, Q.-Y.; Li, Z.; Zhang, W.-H.; Zhang, Z.-C.; Zhang, J.-S.; Li, W.; Ding, H.; Ou, Y.-B.; Deng, P.; Chang, K.; et al. Interface-induced high-temperature superconductivity in single unit-cell FeSe films on $\mathrm{SrTiO}_{3}$. Chin. Phys. Lett. 2012, 29, 037402. [CrossRef]

2. Li, F.; Ding, H.; Tang, C.; Peng, J.; Zhang, Q.; Zhang, W.; Zhou, G.; Zhang, D.; Song, C.-L.; He, K.; et al. Interface-enhanced high-temperature superconductivity in single-unit-cell $\mathrm{FeTe}_{1-x} \mathrm{Se}_{x}$ films onSrTiO ${ }_{3}$. Phys. Rev. B 2015, 91, 220503(R). [CrossRef]

3. Lee, J.J.; Schmitt, F.T.; Moore, R.G.; Johnston, S.; Cui, Y.T.; Li, W.; Yi, M.; Liu, Z.K.; Hashimoto, M.; Zhang, Y.; et al. Interfacial mode coupling as the origin of the enhancement of $\mathrm{T}(\mathrm{c})$ in FeSe films on $\mathrm{SrTiO}_{3}$. Nature 2014, 515, 245-248. [CrossRef]

4. Wang, D.; Kong, L.; Fan, P.; Chen, H.; Zhu, S.; Liu, W.; Cao, L.; Sun, Y.; Du, S.; Schneeloch, J.; et al. Evidence for Majorana bound states in an iron-based superconductor. Science 2018, 362, 333-335. [CrossRef] [PubMed]

5. Zhang, P.; Yaji, K.; Hashimoto, T.; Ota, Y.; Kondo, T.; Okazaki, K.; Wang, Z.J.; Wen, J.S.; Gu, G.D.; Ding, H.; et al. Observation of topological superconductivity on the surface of an iron-based superconductor. Science 2018, 360, 182. [CrossRef]

6. Xu, G.; Lian, B.; Tang, P.; Qi, X.L.; Zhang, S.C. Topological superconductivity on the surface of Fe-based superconductors. Phys. Rev. Lett. 2016, 117, 047001. [CrossRef]

7. Jiang, D.; Pan, Y.; Wang, S.; Lin, Y.; Holland, C.M.; Kirtley, J.R.; Chen, X.; Zhao, J.; Chen, L.; Yin, S.; et al. Observation of robust edge superconductivity in $\mathrm{Fe}(\mathrm{Se}, \mathrm{Te})$ under strong magnetic perturbation. Sci. Bull. 2021, 66, 425-432. [CrossRef]

8. Enayat, M.; Sun, Z.X.; Singh, U.R.; Aluru, R.; Schmaus, S.; Yaresko, A.; Liu, Y.; Lin, C.T.; Tsurkan, V.; Loidl, A.; et al. Real-space imaging of the atomic-scale magnetic structure of $\mathrm{Fe}_{1+\mathrm{y}} \mathrm{Te}$. Science 2014, 345, 653-656. [CrossRef] [PubMed]

9. Trainer, C.; Yim, C.M.; Heil, C.; Giustino, F.; Croitori, D.; Tsurkan, V.; Loidl, A.; Rodriguez, E.E.; Stock, C.; Wahl, P. Manipulating surface magnetic order in iron telluride. Sci. Adv. 2019, 5, eaav3478. [CrossRef] [PubMed]

10. Dagotto, E. Colloquium: The unexpected properties of alkali metal iron selenide superconductors. Rev. Mod. Phys. 2013, 85, 849-867. [CrossRef]

11. Nie, Y.F.; Telesca, D.; Budnick, J.I.; Sinkovic, B.; Wells, B.O. Superconductivity induced in iron telluride films by low-temperature oxygen incorporation. Phys. Rev. B 2010, 82, 020508(R). [CrossRef]

12. Zheng, M. Growth and Oxygen Doping of Thin Film FeTe by Molecular Beam Epitaxy. Available online: http://hdl.handle.net/ 2142/46712 (accessed on 15 August 2021).

13. Hu, H.; Kwon, J.-H.; Zheng, M.; Zhang, C.; Greene, L.H.; Eckstein, J.N.; Zuo, J.-M. Impact of interstitial oxygen on the electronic and magnetic structure in superconducting $\mathrm{Fe}_{1+y} \mathrm{TeO}_{x}$ thin films. Phys. Rev. B 2014, 90, 180504(R). [CrossRef]

14. Zhao, P.H.; Zhu, H.F.; Tian, Y.J.; Li, D.L.; Ma, L.; Suo, H.L.; Nie, J.C. O ${ }_{2}$ Annealing Induced Superconductivity in FeTe $1-x$ Se $_{x}$ : On the Origin of Superconductivity in FeTe Films. J. Super. Nov. Magn. 2016, 30, 871-876. [CrossRef]

15. Mizuguchi, Y.; Deguchi, K.; Tsuda, S.; Yamaguchi, T.; Takano, Y. Evolution of superconductivity by oxygen annealing in FeTe ${ }_{0.8} \mathrm{~S}_{0.2}$. Europhys. Lett. 2010, 90. [CrossRef]

16. Hu, H.; Zuo, J.-M.; Zheng, M.; Eckstein, J.N.; Park, W.K.; Greene, L.H.; Wen, J.; Xu, Z.; Lin, Z.; Li, Q.; et al. Structure of the oxygen-annealed chalcogenide superconductor $\mathrm{Fe}_{1.08} \mathrm{Te}_{0.55} \mathrm{Se}_{0.45} \mathrm{O}_{\mathrm{x}}$. Phys. Rev. B 2012, 85, 064504. [CrossRef]

17. Su, T.S.; Yin, Y.W.; Teng, M.L.; Gong, Z.Z.; Zhang, M.J.; Li, X.G. Effect of carrier density and valence states on superconductivity of oxygen annealed $\mathrm{Fe}_{1.06} \mathrm{Te}_{0.6} \mathrm{Se}_{0.4}$ single crystals. J. Appl. Phys. 2013, 114. [CrossRef]

18. Sun, Y.; Tsuchiya, Y.; Taen, T.; Yamada, T.; Pyon, S.; Sugimoto, A.; Ekino, T.; Shi, Z.; Tamegai, T. Dynamics and mechanism of oxygen annealing in $\mathrm{Fe}_{1+\mathrm{y}} \mathrm{Te}_{0.6} \mathrm{Se}_{0.4}$ single crystal. Sci. Rep. 2014, 4, 4585. [CrossRef]

19. Yamazaki, T.; Sakurai, T.; Yaguchi, H. Size Dependence of oxygen-annealing effects on superconductivity of $\mathrm{Fe}_{1+\mathrm{y}} \mathrm{Te}_{1-\mathrm{x}} \mathrm{S}_{\mathrm{x}}$. J. Phys. Soc. Jpn. 2016, 85, 114712. [CrossRef]

20. Dong, L.; Zhao, H.; Zeljkovic, I.; Wilson, S.D.; Harter, J.W. Bulk superconductivity in $\mathrm{FeTe}_{1-\mathrm{x}} \mathrm{Se}_{\mathrm{x}}$ via physicochemical pumping of excess iron. Phys. Rev. Mater. 2019, 3, 114801. [CrossRef]

21. Ru, H.; Lin, Y.-S.; Chen, Y.-C.; Feng, Y.; Wang, Y.-H. Observation of two-level critical state in the superconducting FeTe thin films. Chin. Phys. Lett. 2019, 36, 077402. [CrossRef]

22. Kawasaki, Y.; Deguchi, K.; Demura, S.; Watanabe, T.; Okazaki, H.; Ozaki, T.; Yamaguchi, T.; Takeya, H.; Takano, Y. Phase diagram and oxygen annealing effect of $\mathrm{FeTe}_{1-\mathrm{x}} \mathrm{Se}_{\mathrm{x}}$ iron-based superconductor. Solid State Commun. 2012, 152, 1135-1138. [CrossRef] 
23. Si, W.; Jie, Q.; Wu, L.; Zhou, J.; Gu, G.; Johnson, P.D.; Li, Q. Superconductivity in epitaxial thin films of Fe 1.08 Te:O $_{x}$. Phys. Rev. B 2010, 81, 092506. [CrossRef]

24. Horcas, I.; Fernandez, R.; Gomez-Rodriguez, J.M.; Colchero, J.; Gomez-Herrero, J.; Baro, A.M. WSXM: A software for scanning probe microscopy and a tool for nanotechnology. Rev. Sci. Instrum. 2007, 78, 013705. [CrossRef] [PubMed]

25. Zhang, W.-H.; Sun, Y.; Zhang, J.-S.; Li, F.-S.; Guo, M.-H.; Zhao, Y.-F.; Zhang, H.-M.; Peng, J.-P.; Xing, Y.; Wang, H.-C.; et al. Direct Observation of high-temperature superconductivity in one-unit-cell FeSe films. Chin. Phys. Lett. 2014, 31, 017401. [CrossRef]

26. Liang, J.; Zhang, Y.J.; Yao, X.; Li, H.; Li, Z.-X.; Wang, J.; Chen, Y.; Sou, I.K. Studies on the origin of the interfacial superconductivity of $\mathrm{Sb}_{2} \mathrm{Te}_{3} / \mathrm{Fe}_{1+\mathrm{y}} \mathrm{Te}$ heterostructures. Proc. Natl. Acad. Sci. USA 2020, 117, 221-227. [CrossRef] [PubMed]

27. Telesca, D.; Nie, Y.; Budnick, J.I.; Wells, B.O.; Sinkovic, B. Impact of valence states on the superconductivity of iron telluride and iron selenide films with incorporated oxygen. Phys. Rev. B 2012, 85, 214517. [CrossRef]

28. Zhang, Z.T.; Yang, Z.R.; Lu, W.J.; Chen, X.L.; Li, L.; Sun, Y.P.; Xi, C.Y.; Ling, L.S.; Zhang, C.J.; Pi, L.; et al. Superconductivity in $\mathrm{Fe}_{1.05} \mathrm{Te}: \mathrm{O}_{\mathrm{x}}$ single crystals. Phys. Rev. B 2013, 88, 214511. [CrossRef]

29. Smith, N.; Gelting, D.; Basaran, A.C.; Schofield, M.; Schuller, I.K.; Gajdardziska-Josifovska, M.; Guptasarma, P. Effects of oxygen annealing on single crystal iron telluride. Phys. C Supercond. 2019, 567, 1253400. [CrossRef]

30. Zhang, Z.; Cai, M.; Li, R.; Meng, F.; Zhang, Q.; Gu, L.; Ye, Z.; Xu, G.; Fu, Y.-S.; Zhang, W. Controllable synthesis and electronic structure characterization of multiple phases of iron telluride thin films. Phys. Rev. Mater. 2020, 4, 125003. [CrossRef]

31. Song, Y.; Ying, T.; Chen, X.; Han, X.; Huang, Y.; Wu, X.; Schnyder, A.-P.; Guo, J.; Chen, X. Enhancement of superconductivity in hole-doped $\mathrm{CsV}_{3} \mathrm{Sb}_{5}$ thin films. arXiv 2021, arXiv:2105.09898v1.

32. Qn, H.; Guo, B.; Wang, L.; Zhang, M.; Xu, B.; Shi, K.; Pan, T.; Zhou, L.; Chen, J.; Qu, Y.; et al. Superconductivity in singlequintuple-layer $\mathrm{Bi}_{2} \mathrm{Te}_{3}$ grown on epitaxial FeTe. Nano Lett. 2020, 20, 3160-3168. [CrossRef] [PubMed]

33. Owada, K.; Nakayama, K.; Tsubono, R.; Shigekawa, K.; Sugawara, K.; Takahashi, T.; Sato, T. Electronic structure of a Bi $2 \mathrm{Te}_{3} / \mathrm{FeTe}$ heterostructure: Implications for unconventional superconductivity. Phys. Rev. B 2019, 100, 064518. [CrossRef]

34. Guo, B.; Shi, K.-G.; Qin, H.-L.; Zhou, L.; Chen, W.-Q.; Ye, F.; Mei, J.-W.; He, H.-T.; Pan, T.-L.; Wang, G. Evidence for topological superconductivity: Topological edge states in $\mathrm{Bi}_{2} \mathrm{Te}_{3} / \mathrm{FeTe}$ heterostructure. Chin. Phys. B 2020, 29, 097403. [CrossRef]

35. Yuan, Y.; Wang, X.; Song, C.; Wang, L.; He, K.; Ma, X.; Yao, H.; Li, W.; Xue, Q.-K. Observation of coulomb gap and enhanced superconducting gap in nano-sized $\mathrm{Pb}$ islands grown on $\mathrm{SrTiO}_{3}$. Chin. Phys. Lett. 2020, 37, 017402. [CrossRef] 